\title{
V-sis Platelet Derived Growth Factor, Beta Polypeptide
}

National Cancer Institute

\section{Source}

National Cancer Institute. v-sis Platelet Derived Growth Factor, Beta Polypeptide. NCI

Thesaurus. Code C20077.

V-sis Platelet Derived Growth Factor beta polypeptide is the viral homologue of PDGFB.

Viral oncogenes originate from normal cellular genes by recombination between a parent nontransforming virus and host cellular DNA; the cellular genes often code for important functions in cell growth or tissue differentiation. PDGF is the major serum polypeptide mitogen for cells of mesenchymal origin and has two dissimilar subunits, A and B. The B chain has homology to SIS and is alone sufficient for mitogenesis. Most proliferating cells are programmed to undergo apoptosis unless specific survival signals are provided. PDGF promotes proliferation and inhibits apoptosis by activating the RAS/PIK3/AKT 1/IKK/NFKB1 pathway, inducing putative anti-apoptotic genes. (from OMIM 190040 and $\mathrm{NCl}$ ) 